\title{
A Generalized Interpolation Algorithm
}

\author{
By A. C. R. Newbery
}

\begin{abstract}
An interpolation algorithm is derived, which will construct an $(n+1)$-point interpolant based on any sequence of interpolatory functions that can be defined by a three-term linear recursion. By suitable parameter choice, a single algorithm can be made to interpolate in terms of the classical polynomial sequences or in terms of trigonometric or hyperbolic series etc. An analysis of truncation error is included.
\end{abstract}

Given $n+1$ points $\left(x_{i}, f_{i}\right)$ with the $x_{i}$ distinct, and given $n+1$ functions $\phi_{i}(x)$, the interpolation problem consists in calculating $n+1$ weights $w_{i}$ such that

$$
f_{i}=\sum_{i=0}^{n} w_{i} \phi_{i}\left(x_{i}\right), \quad i=0,1, \cdots, n .
$$

Apart from the familiar Lagrangian case, where $\phi_{i}(x)=x^{i}$, the commonest cases involve basis functions $\phi_{i}$ which obey a three-term recursion of the form

$$
\phi_{i+1}=\left(g(x)-\alpha_{j}\right) \phi_{i}-\beta_{j} \phi_{j-1}, \quad j=0,1, \cdots,
$$

where the $\alpha_{i}, \beta_{i}$ are given scalars, $g(x)$ and $\phi_{0}(x)$ are given functions, and $\phi_{-1}(x) \equiv 0$. This case includes direct interpolation in terms of the classical polynomials of Legendre, Chebyshev, etc.; it also includes interpolation in terms of sine and cosine polynomials and their hyperbolic analogs. We now wish to construct an algorithm for which the input data are vectors with components $x_{i}, f_{i}, \alpha_{i}, \beta_{i}, \phi_{0}\left(x_{i}\right), g\left(x_{i}\right)$ and the output vector has components $w_{i}$ in accordance with (1), (2). The subscripts $i, j$ run through the range $[0, n]$ with $\beta_{0}=0$.

The development of the algorithm will follow the same pattern as used in [1] for the special case of sine series interpolation. First, we develop a sequence of functions $\left\{\pi_{k}(x)\right\}, k=1,2, \cdots$, with the properties that (a) $\pi_{k}(x)$ is a linear combination of the $\phi_{i}(x)$ for $j \leqq k$ with the coefficient of $\phi_{k}$ being unity, (b) $\pi_{k}\left(x_{i}\right)=0$ for $i<k$ and for no other subscript $i$. In order to meet these requirements, we set $\pi_{1}(x)=$ $\phi_{1}-\left(g\left(x_{0}\right)-\alpha_{0}\right) \phi_{0}=\left(g(x)-g\left(x_{0}\right)\right) \phi_{0}$. (It follows from (2) that these two formulations are equivalent.) Subsequently, $\pi_{k+1}(x)=\left(g(x)-g\left(x_{k}\right)\right) \pi_{k}(x)$. The fact that (2) defines a way of expressing $g \phi_{i}$ as a linear combination of $\phi_{i+1}, \phi_{i}, \phi_{i-1}$ implies that $\pi_{k+1}$ is of the required form. At this stage, therefore, we may conclude that for each $k$ there exist coefficients $p_{i}(k)$ such that

$$
\pi_{k}(x)=\prod_{i=0}^{k-1}\left(g(x)-g\left(x_{i}\right)\right) \phi_{0}(x)=\sum_{i=0}^{k} p_{i} \phi_{i}(x), \quad p_{k}=1 .
$$

In order to meet the requirement that $\pi_{k}(x)$ should not vanish at any node $x_{i}$ other than those for which $i<k$, it is sufficient to impose the restrictions that $\phi_{0}\left(x_{i}\right) \neq 0$

Received July 23, 1970, revised December 14, 1970.

AMS 1969 subject classifications. Primary 6520, 6580; Secondary 4130.

Key words and phrases. Interpolation, osculatory interpolation, Bürmann series. 
at all nodes and $g\left(x_{i}\right) \neq g\left(x_{i}\right)$ for all distinct nodes $x_{i}, x_{i}$. These are the minimum restrictions under which the interpolant is constructible. Let $L_{k}(x)$ denote a linear combination of the basis functions $\phi_{0}, \phi_{1}, \cdots, \phi_{k}$ such that $L_{k}\left(x_{i}\right)=f_{i}$ for $i \leqq k$. The $L_{k}$ are constructible, as is easily verified, by the following recursion:

$$
\begin{aligned}
L_{0}(x) & =f_{0} \phi_{0}(x) / \phi_{0}\left(x_{0}\right), \quad b_{k}=\left[f_{k+1}-L_{k}\left(x_{k+1}\right)\right] / \pi_{k+1}\left(x_{k+1}\right), \\
L_{k+1}(x) & =L_{k}(x)+b_{k} \pi_{k+1}(x), \quad k=0,1, \cdots, n-1 .
\end{aligned}
$$

In executing this algorithm, it is recommended that the evaluation of $\pi_{k+1}\left(x_{k+1}\right)$ in the expression for $b_{k}$ should be performed using the product form (3), while one would need to use the summation form in (3) when deriving the $\phi$-polynomial form of $L_{k+1}$ from that of $L_{k}$. This summation form has been stated explicitly for $\pi_{1}$; thereafter, each one may be computed as follows: Let $\pi_{k}=\sum_{i=0}^{k} p_{i} \phi_{i}(x)$ and $\pi_{k+1}=$ $\sum_{j=0}^{k+1} p_{i}^{\prime} \phi_{j}(x)$, then

$$
p_{i}^{\prime}=\left(\alpha_{i}-g\left(x_{k}\right)\right) p_{i}+p_{i-1}+\beta_{i+1} p_{i+1}, \quad j=0,1, \cdots, k+1,
$$

where the undefined coefficients $p_{k-1}, p_{k+1}$, and $p_{k+2}$ are set equal to zero. The evaluation of $L_{k}(x) \equiv \sum_{i=0}^{k} q_{i} \phi_{j}(x)$ for $x=x_{k+1}$, which is needed to compute $b_{k}$ in (4), may be done in either of two ways: First, if a complete matrix of quantities $\phi_{i}\left(x_{i}\right)$ is available, then the computation is simply a scalar product of two $(k+1)$-dimensional vectors. On the other hand, if this matrix is not available and is not needed for other purposes, it would be a waste of time and storage to compute it specifically for this job. Instead, one should recognize that evaluating $L_{k}$ is formally equivalent to evaluating a weighted sum of orthogonal polynomials, and a suitable algorithm adapted from [3, p. 70] is:

$$
\begin{aligned}
& t_{k}=q_{k}, \quad t_{k-1}=q_{k-1}+t_{k}\left(g(x)-\alpha_{k-1}\right), \\
& t_{r}=q_{r}+t_{r+1}\left(g(x)-\alpha_{r}\right)-\beta_{r+1} t_{r+2}, \quad r=k-2, k-3, \ldots, 0 .
\end{aligned}
$$

The value of $L_{k}(x)$ is then $t_{0} \phi_{0}(x)$.

In order to derive an error term associated with our $(n+1)$-point generalized interpolation algorithm, it will be necessary to make some more restrictive assumptions on the nature of $g(x), \phi_{0}(x)$. Let $I$ be the continuous interval on the $x$-axis over which interpolation is required; thus $I$ includes all the $x_{i}$ and any other $x$-value at which the interpolant may be evaluated. We require that $f, g, \phi_{0}$ be differentiable at least $n+1$ times in $I$ and that neither $g^{\prime}$ nor $\phi_{0}$ vanishes in $I$. It will be observed that these conditions imply the weaker conditions that were earlier stated to be necessary and sufficient for the construction of the interpolant. The $(n+1)$-point interpolant $L_{n}(x)$ is therefore constructible, and by examination of algorithm (6), we see that $L_{n}(x) \equiv P_{n}(g) \phi_{0}(x)$, where $P_{n}$ is an algebraic polynomial of degree $n$ in the variable $g=g(x)$. Writing $g_{i}=g\left(x_{i}\right)$, we have therefore implicitly constructed a polynomial $P_{n}$ such that, at all nodes $x_{i}$,

$$
f\left(x_{i}\right)=L_{n}\left(x_{i}\right)=P_{n}\left(g_{i}\right) \phi_{0}\left(x_{i}\right) .
$$

Equivalently, writing $g(x)=z$, we have constructed a polynomial $P_{n}$ such that

$$
P_{n}(z)=f\left(g^{-1}(z)\right) / \phi_{0}\left(g^{-1}(z)\right)
$$

at every point $z_{i}=g\left(x_{i}\right)$. The fact that this is a well-defined expression follows from 
our assumption that $g^{\prime}, \phi_{0}$ do not vanish in $I$. The expression for the internodal error $E(z)$ in (7) is the standard Lagrangian error term [2, p. 63]:

$$
\begin{aligned}
E(z) & =f\left(g^{-1}(z)\right) / \phi_{0}\left(g^{-1}(z)\right)-P_{n}(z) \\
& =\frac{1}{(n+1) !} \prod_{i=0}^{n}\left(z-z_{i}\right)\left(\frac{d}{d z}\right)^{n+1}\left[f\left(g^{-1}(z)\right) / \phi_{0}\left(g^{-1}(z)\right)\right]_{z-5},
\end{aligned}
$$

where $g^{-1}(\zeta) \in I$.

In order to investigate the differential expression in (8), we define $Q_{r}(x)=$ $(d / d z)^{r}\left[f(x) / \phi_{0}(x)\right]$. We have $Q_{0}=f(x) / \phi_{0}(x), Q_{1}=\left(\phi_{0} f^{\prime}-\phi_{0}^{\prime} f\right) / g^{\prime} \phi_{0}^{2}$. If we let $Q_{r}=N_{r} / g^{\prime 2 r-1} \phi_{0}^{r+1}, r=1,2 \cdots$, then

$$
N_{r+1}=N_{r}^{\prime} g^{\prime} \phi_{0}-N_{r}(2 r-1) g^{\prime \prime} \phi_{0}+(r+1) g^{\prime} \phi_{0}^{\prime} .
$$

The differential expression $N$, is linear in $f$ but its coefficients involve nonlinear combinations of $\phi_{0}, g^{\prime}$ and their derivatives. We conclude that $Q_{n+1}$, which is the differential expression in (8), is $N_{n+1} /\left(g^{2 n+1} \phi_{0}^{n+2}\right)$. Actually, the error in our interpolation is not $E(z)$ but $\widetilde{E}(x)$, where

$$
\tilde{E}(x)=\phi_{0}(x) E(z)=\frac{\phi_{0}(x)}{(n+1) !} \prod_{i=0}^{n}\left(g(x)-g\left(x_{i}\right)\right) N_{n+1} /\left[{g^{\prime 2 n+1}}^{2 n)} \phi_{0}^{n+2}(\eta)\right],
$$

where $N_{n+1}$ is evaluated at some point $\eta$ in $I$. Although the structure of $N_{n+1}$ is too complicated to permit any clear practical insights, there is still something to be learned from the form of the error term (9). At first, it appears that if $g^{\prime}$ or $\phi_{0}$ becomes small anywhere in $I$, then large errors are to be expected; however, it may be noted that if $\phi_{0}$ and $g$ are multiplied by constants $p, q$, the error remains invariant. This can be seen by following our derivation, and it is also evident geometrically. We conclude that large errors may be expected if $g^{\prime}$ is small relative to $g$ or relative to its higher derivatives (which are implicit in $N_{n+1}$ ) anywhere in $I$; similarly, the smallness of $\phi_{0}$ relative to its derivatives has to be seen as a danger signal. For instance, if one is constructing a cosine series interpolant, one normally takes $\phi_{0}=1, g=2 \cos x$. If the interval $I$ contains points $\eta$ such that $\sin \eta$ is small, then we run into one of the above mentioned problems. If we try to construct a sine series interpolant, then we encounter both the above problems.

So far we have assumed that we were required to construct an explicit approximation to $f(x)$ in terms of the basis functions $\phi_{i}$. In the simpler case where all that is needed is an algorithm for pointwise interpolation, the simplest procedure would be to use the fact that, at nodes $x_{i}, f\left(x_{i}\right)=P_{n}\left(g_{i}\right) \phi_{0}\left(x_{i}\right)$; when interpolating, we assume that the same relation holds, at least approximately, also at nonnodal points. At every node $x_{i}$, we compute $g_{i}$ and $\tilde{f}_{i}=f\left(x_{i}\right) / \phi_{0}\left(x_{i}\right)$. Then, implicitly or explicitly, we construct the algebraic polynomial $P_{n}(g)$ such that $P_{n}\left(g_{i}\right)=f_{i}$; then we assume that, at any nonnodal point $x, P_{n}(g(x))=\tilde{f}(x)=f(x) / \phi_{0}(x)$. For the given $x$, we compute $g(x)$ and evaluate $P_{n}(g)$ for this argument, using any reputable form of polynomial interpolation. This yields an interpolated value for $\tilde{f}(x)$, which must be multiplied by $\phi_{0}(x)$ to yield the interpolated value of $f(x)$. This procedure, which is mathematically equivalent to the construction and evaluation of $L_{n}(x)$, will generally be considered computationally preferable when pointwise interpolation is all that is required. The user can apply the efficient and well-understood algorithms for poly- 
nomial interpolation; he only needs to pre-edit the data and post-edit the output.

It is also possible to write generalized algorithms of this type for osculatory and other confluent cases; however, we do encounter difficulties which do not arise in the distinct-node case. We will investigate here only the Taylor-series analog, in which the function $f(x)$ is specified by its ordinate and successive derivatives at a single node $x_{1}$. The product form of (3) is replaced by

$$
\pi_{k}(x)=\left(g(x)-g\left(x_{1}\right)\right)^{k} \phi_{0}(x) .
$$

$L_{k}(x)$ is defined as that linear combination of $\phi_{0}, \phi_{1}, \cdots, \phi_{k}$ which matches derivatives through the $k$ th with $f(x)$ at $x_{1}$. It is defined as in (4), except that

$$
b_{k}=\left(f^{(k+1)}-L_{k}^{(k+1)}\left(x_{1}\right)\right) / \pi_{k+1}^{(k+1)}\left(x_{1}\right) .
$$

The denominator in ( $\left.4^{\prime}\right)$ simplifies to $(k+1) ! g^{\prime k+1}\left(x_{1}\right) \phi_{0}\left(x_{1}\right)$, but we have no simple way, in general, of evaluating the $(k+1)$ th derivative of $L_{k}$ at $x_{1}$. There will be cases where this is a trivial problem, as when $g(x), \phi_{0}(x), \alpha_{i}, \beta_{i}$ are chosen to yield a sine or cosine series, but there appears to be no simple way of proceeding in the absence of such special information. A more serious objection is that the algorithm may fail to solve solvable problems of confluent type. For instance, it is a well-posed problem to construct a cosine series approximation to an even function whose Maclaurin expansion is known; however, the expression (4') would be undefined because $g(x)=2 \cos x$ and $g^{\prime}(0)=0$. It is possible to bypass this difficulty by writing $\phi_{0}(x)=1, g(x)=2 \sin (x / 2)$. This generates an expansion in the space of $1, \sin (x / 2)$, $\cos x, \sin (3 x / 2), \cos 2 x, \cdots$, and since all odd-order derivatives of the function are zero, so will all coefficients of the sine terms be zero, and we therefore have the required cosine expansion.

It may be noted that when $\phi_{0}(x) \equiv 1$, the confluent form of our algorithm is mathematically equivalent to the Bürmann series [2, p. 25], although the computational pattern has been simplified and made more adaptable for automatic computation.

In summary, it seems that, in the discrete-node case, we can achieve a high level of versatility without great degradation of performance. This versatility should be valuable in situations where some searching or experimentation is needed before deciding on the exact form of interpolant. Where confluent cases are concerned, the coverage is less good, and ad hoc remedies are sometimes needed; nevertheless, there are probably useful applications in this area too.

University of Kentucky

Lexington, Kentucky 40506

1. A. C. R. NewBery, "Interpolation by algebraic and trigonometric polynomials," Math. Comp., v. 20, 1966, pp. 597-599. MR 34 \#3752.

2. F. B. Hildebrand, Introduction to Numerical Analysis, McGraw-Hill, New York, 1956. MR 17,788.

3. J. HART et AL., Computer Approximations, Wiley, New York, 1968. 